\title{
Methylation status of WWOX gene promoter CpG islands in epithelial ovarian cancer and its clinical significance
}

\author{
HONGCHAO YAN and JIEYUN SUN \\ Department of Obstetrics and Gynecology, The Affiliated Hospital of Xuzhou Medical College, \\ Xuzhou, Jiangsu 221002, P.R. China
}

Received December 2, 2012; Accepted January 24, 2013

DOI: $10.3892 /$ br.2013.86

\begin{abstract}
WW domain-containing oxidoreductase (WWOX) is a newly identified tumor suppressor gene that is associated with abnormal DNA methylation. The aim of this study was to evaluate the methylation status of $\mathrm{CpG}$ islands in the WWOX gene promoter region in cases of epithelial ovarian cancer and explore the correlation between the methylation status of the WWOX gene CpG islands and clinicopathological indices in patients with epithelial ovarian cancer. The methylation status of the WWOX gene CpG island was evaluated by methylation-specific polymerase chain reaction (MSP) in 48 patients with epithelial ovarian cancer, 18 patients with borderline epithelial ovarian tumors, 26 patients with epithelial benign tumors and 33 patients with normal ovarian tissues. Results showed that the rates of $\mathrm{CpG}$ island methylation in the WWOX gene promoter region in epithelial ovarian cancer tissues, borderline ovarian tumor tissues and benign ovarian tumor tissues were 43.75, 26.32 and $3.84 \%$, respectively. The WWOX gene $\mathrm{CpG}$ islands were not methylated in normal ovarian tissues. The rate of $\mathrm{CpG}$ island methylation in epithelial ovarian cancer tissues was higher than that of other ovarian tissues and these differences were found to be statistically significant $(\mathrm{P}<0.01)$. The rate of $\mathrm{CpG}$ island methylation in the WWOX gene promoter region in late-stage (stage III and IV) epithelial ovarian cancer tissues was higher than that of early-stage (stage I and II) epithelial ovarian cancer tissues, and these differences were found to be statistically significant $(\mathrm{P}<0.05)$. In conclusion, epithelial ovarian cancer tissues showed $\mathrm{CpG}$ island hypermethylation in the WWOX gene promoter region, which may be an important mechanism leading to WWOX gene inactivation. Atypical methylation of WWOX gene is associated with the formation and progression of epithelial ovarian cancer, rendering it a potentially
\end{abstract}

Correspondence to: Dr Hongchao Yan, Department of Obstetrics and Gynecology, The Affiliated Hospital of Xuzhou Medical College, 99 Huaihai West Road, Xuzhou, Jiangsu 221002, P.R. China E-mail: hongchaoyan@hotmail.com

Key words: epithelial ovarian cancer, WW domain-containing oxidoreductase gene, $\mathrm{CpG}$ island, DNA methylation important indicator in the early diagnosis and prognosis of epithelial ovarian cancer.

\section{Introduction}

Epigenetic changes do not alter the nucleotide gene sequence, however, alteration of the phenotype leads to heritable changes in gene expression. The main types of epigenetic changes are DNA methylation, chromatin restructuring, histone modification and RNA editing. In mammals, DNA methylation is the most common natural chemical modification and is important in adjusting gene expression and maintaining the normal differentiation of cells. Recent studies have demonstrated that abnormal DNA methylation is important in the development of tumor progression (1). Abnormal DNA methylation mainly occurs in the gene promoter region ( $\mathrm{CpG}$ island); the $\mathrm{CpG}$ island in gene promoter region of normal tissue is unmethylated. Thus, when abnormal methylation occurs in the 5'-cytosine of the CpG island sequence, it can lead to suppression of gene transcription. This, in turn, leads to the inhibition of the anticancer gene, resulting in tumor formation $(2,3)$. The WW domain-containing oxidoreductase (WWOX) gene is a newly identified tumor suppressor gene that crosses the chromosomal fragile site, FRA16D (4). Abnormal expression of the WWOX gene in bladder, breast, prostate and stomach cancer, as well as other tumors, is associated with abnormal DNA methylation (5-7). The aim of this study was to examine the association between the aberrant methylation status of WWOX gene promoter $\mathrm{CpG}$ islands and epithelial ovarian cancer.

\section{Materials and methods}

Patient samples. Between October 2009 and June 2011, we collected 48 surgically-resected epithelial ovarian cancer tissues (serous cystadenocarcinoma, 29; mucinous cystadenocarcinoma, 19), 18 borderline epithelial ovarian tumors, 26 epithelial benign tumors and 33 normal ovarian tissues (due to myoma of the uterus, these patients underwent hysterectomy and bilateral salpingo-oophorectomy and subsequently the tissues were pathologically confirmed as normal ovarian tissues) obtained from patients in the Affiliated Hospital of Xuzhou Medical College, Xuzhou Maternity and Child Centers and Xuzhou Tumor Hospital. Fresh surgical speci- 
Table I. Methylation status of WWOX gene promoter CpG island in different ovarian tissue types.

\begin{tabular}{lccccc}
\hline Tissue type & Positive no. & Negative no. & Methylation rate (\%) & Test method & P-value \\
\hline Epithelial ovarian cancer & 21 & 27 & $43.75^{\mathrm{a}}$ & $\chi^{2}=28.243$ & $<0.01$ \\
Borderline epithelial ovarian tumor & 5 & 13 & $27.78^{\mathrm{b}}$ & Fisher's exact test & \\
Benign ovarian epithelial tumor & 1 & 25 & $3.84^{\mathrm{b}}$ & Fisher's exact test & \\
Normal ovarian tissue & 0 & 33 & $0.00^{\mathrm{b}}$ & Fisher's exact test &
\end{tabular}

Borderline epithelial tumors and benign epithelial tumors, $\mathrm{P}<0.05$. Borderline epithelial tumors compared with normal ovarian tissue, $\mathrm{P}<0.01$. Benign epithelial tumors compared with normal ovarian tissue, $P>0.05$. ${ }^{\text {a The }} \chi^{2}$ test was used to compare ovarian epithelial cancer and other ovarian tissue. ${ }^{\mathrm{b}} \mathrm{A}$ comparison of borderline epithelial ovarian tumors, ovarian benign epithelial tumors and normal ovarian tissue was carried out. WWOX, WW domain-containing oxidoreductase.

mens were cultured using a common method, then stored at $-80^{\circ} \mathrm{C}$ to investigate the methylation status of WWOX gene $\mathrm{CpG}$ islands.

The study was approved by the Ethics Committee of Xuzhou Medical College, Xuzhou, Jiangsu, China. Informed consent was obtained from the patients prior to the study.

Experimental reagents and primers. Reagents used in the study included the Ezup column animal genome DNA extraction kit (Shanghai Shengong Bioengineering Co., Ltd., Shanghai, China), EpiTect Bisulfite kit (used for DNA modification; Qiagen, Hilden, Germany) and the EpiTect methylation-specific PCR (MSP) kit [used for MSP; Qiagen ]. Primers were purchased from Invitrogen (Shanghai, China). The methylation primer sequence of the WWOX gene was: forward, 5'-TATGGGCGTCGTTTTTTTAGTT-3'; and reverse, 5'-CAATCTCCGCAATATCGCGACA-3' (product length, $347 \mathrm{bp}$ ). The unmethylated primer sequence used was: forward, 5'-TATGGGTGTTGTTTTTTTAGTT-3'; and reverse, 5'-CAATCTCCACAATATCAC AACA-3' (product length, $347 \mathrm{bp})$.

DNA extraction. Approximately $30 \mathrm{mg}$ tissue specimen was obtained from the surgically resected samples, i.e., epithelial ovarian cancer, borderline epithelial ovarian tumor, benign ovarian epithelial tumor and normal ovary tissue samples. Samples were triturated in liquid nitrogen and DNA was extracted using an Ezup column animal genome DNA extraction kit (Shanghai Shengong Bioengineering Co., Ltd.), according to the manufacturer's instructions. The extracted DNA was subjected to agarose gel electrophoresis to obtain DNA bands and was visualized under light microscopy. DNA purity was estimated using an ultraviolet spectrophotometer. The adsorption (A) value was measured as A260/A280:1.8-2.0.

Modification and purification of DNA sulfite. Reagent was prepared according to the manufacturer's instructions (EpiTect Bisulfite kit, Qiagen). DNA (15 $\mu \mathrm{l})$ was added to RNase-free water, until a total volume of $20 \mu \mathrm{l}$ was obtained, and then to a bisulfite mix (85 $\mu 1$ dissolved Bisulfite Mix and $35 \mu 1$ DNA Protect buffer; EpiTect Bisulfite kit) and amplified using polymerase chain reaction (PCR) according to the manufacturer's instructions. The modified DNA was moved into an EpiTect centrifugal column, and underwent maximum speed

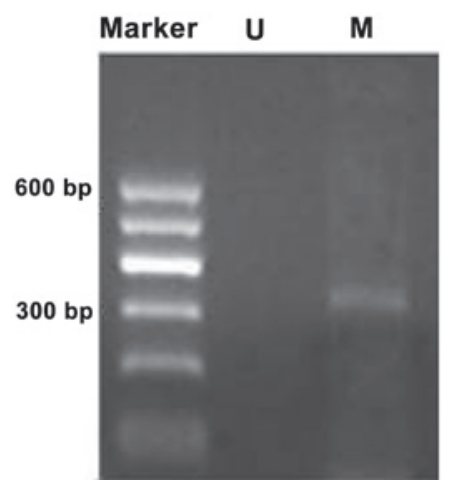

Figure 1. Methylation status of the WW domain-containing oxidoreductase gene promoter $\mathrm{CpG}$ islands in epithelial ovarian cancer was completely methylated. M, methylated band; U, unmethylated band.

centrifugation for $1 \mathrm{~min}$. The liquid waste was then discarded and the sulfonation liquid was added, followed by desalination and purification. Finally, $30 \mu \mathrm{l}$ DNA was obtained by washing with Buffer EB.

MSP. MSP was carried out in a total volume of $50 \mu \mathrm{l}$ (EpiTect Master Mix, $25 \mu$ l), with modified DNA (4 $\mu \mathrm{l})$ in RNase-free water. The reaction conditions for MSP were: initial denaturation at $95^{\circ} \mathrm{C}$ for $10 \mathrm{~min}$, followed by $94^{\circ} \mathrm{C}$ for $30 \mathrm{sec} ; 58^{\circ} \mathrm{C}$ for $45 \mathrm{sec} ; 72^{\circ} \mathrm{C}$ for $45 \mathrm{sec}$ for 40 cycles; and $72^{\circ} \mathrm{C}$ for $10 \mathrm{~min}$ for both methylated and unmethylated reactions. The PCR products $(6 \mu \mathrm{l})$ were electrophoresed, verified in $1.5 \%$ agarose gel and observed under UV light. Target gene amplification of methylation primers was considered fully methylated, while that of unmethylated primers was considered partially methylated.

Statistical analysis. Data were analyzed using SPSS 13.0 statistical software. A comparison of the various sample rates and the differences of the methylation rates between the two groups was carried out using $\chi^{2}$ test. Statistical significance was defined as $\mathrm{P}<0.05$.

\section{Results}

Methylation status of WWOX gene promoter CpG islands status in various ovarian tissues. The methylation rate of WWOX gene promoter $\mathrm{CpG}$ islands in epithelial ovarian 
Table II. Association between the methylation rate of WWOX gene promoter CpG islands and the clinicopathological characteristics of epithelial ovarian cancer.

\begin{tabular}{|c|c|c|c|c|c|}
\hline $\begin{array}{l}\text { Clinicopathological } \\
\text { characteristics }\end{array}$ & Positive no. & Negative no. & Methylation rate (\%) & $\chi^{2}$ value & P-value \\
\hline \multicolumn{6}{|l|}{ Age (years) } \\
\hline$>50$ & 13 & 15 & 46.43 & \multirow[t]{2}{*}{0.196} & \multirow[t]{2}{*}{0.658} \\
\hline$\leq 50$ & 8 & 12 & 40.00 & & \\
\hline \multicolumn{6}{|l|}{ Clinical stages } \\
\hline I-II & 7 & 17 & 29.17 & \multirow[t]{2}{*}{4.148} & \multirow[t]{2}{*}{0.042} \\
\hline III-IV & 14 & 10 & 58.33 & & \\
\hline \multicolumn{6}{|l|}{ Pathological grading } \\
\hline G1 & 5 & 9 & 35.71 & \multirow{3}{*}{0.602} & \\
\hline $\mathrm{G} 2$ & 9 & 11 & 45.00 & & \\
\hline G3 & 7 & 7 & 50.00 & & 0.740 \\
\hline \multicolumn{6}{|l|}{ Pathological type } \\
\hline Serous & 12 & 17 & 41.38 & \multirow[t]{2}{*}{0.167} & \\
\hline Mucinous & 9 & 10 & 47.37 & & 0.683 \\
\hline \multicolumn{6}{|c|}{ Lymph node metastasis } \\
\hline Yes & 6 & 7 & 46.15 & \multirow[t]{2}{*}{0.042} & \\
\hline No & 15 & 20 & 42.86 & & 0.838 \\
\hline \multicolumn{6}{|l|}{ Ascites } \\
\hline Yes & 14 & 16 & 46.67 & \multirow[t]{2}{*}{0.277} & \\
\hline No & 7 & 11 & 38.89 & & 0.599 \\
\hline
\end{tabular}

WWOX, WW domain-containing oxidoreductase.

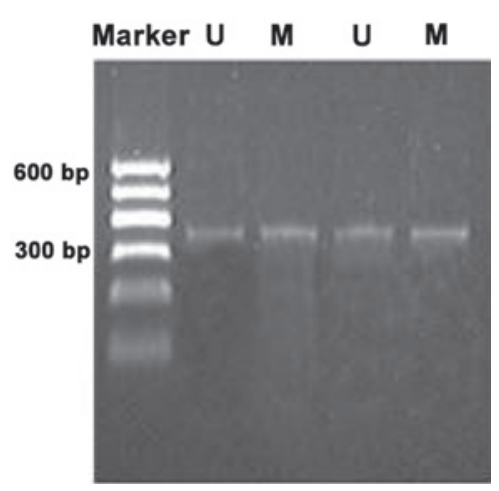

Figure 2. Methylation status of the WW domain-containing oxidoreductase gene promoter $\mathrm{CpG}$ islands in epithelial ovarian cancer was partly methylated. U, unmethylated band; M, methylated band.

cancer, borderline ovarian epithelial tumors and benign ovarian epithelial tumors was found to be $43.75,26.32$ and $3.84 \%$, respectively. The $\mathrm{CpG}$ islands in the WWOX gene in normal ovarian tissues were completely unmethylated (Table I). The rate of $\mathrm{CpG}$ island methylation in epithelial ovarian cancer tissues was higher than that of the remaining three ovarian tissue types, and these differences were found to be statistically significant $(\mathrm{P}<0.01)$. Additionally, the methylation rate of borderline epithelial ovarian tumors was higher than that of benign ovarian epithelial tumors and normal ovarian tissue, respectively (Fisher's exact test; $\mathrm{P}<0.05)$.

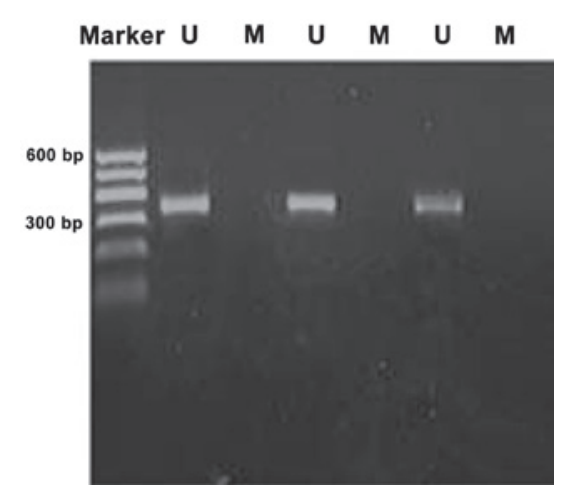

Figure 3. Methylation status of the WW domain-containing oxidoreductase gene promoter $\mathrm{CpG}$ islands in normal ovarian tissue was unmethylated. $\mathrm{M}$, methylation amplification band; $\mathrm{U}$, unmethylated band.

Methylation bands were identified in 21 of 48 epithelial ovarian cancer cases, of which 13 were fully methylated (Fig. 1) and 8 were partly methylated (Fig. 2). Five of 18 borderline epithelial ovarian tumor cases had methylation bands, of which 3 were fully methylated and 2 were partly methylated. One benign ovarian epithelial tumor case was completely methylated. In normal ovarian tissues, only unmethylated bands were amplified (Fig. 3).

Association between the methylation rate of WWOX gene promoter $\mathrm{Cp} G$ island and clinicopathological characteristics of epithelial ovarian cancer. The rate of $\mathrm{CpG}$ island 
methylation in the WWOX gene promoter region in late-stage (stage III and IV) epithelial ovarian cancer tissues was higher than that of early-stage (stage I and II) epithelial ovarian cancer tissues, and these differences were found to be statistically significant $(\mathrm{P}<0.05$; Table II). By contrast, no statistically significant correlation was detected between the methylation rate of the WWOX gene and clinicopathological characteristics such as age, pathologies (serous epithelial and mucinous carcinoma), pathological grading (I, II and III), ascites and lymph node metastasis.

\section{Discussion}

Epithelial ovarian cancer is a common malignancy in females. It is characterized by a complex etiology, incidence of concealment and rapid progression. The mortality rate is the highest of three malignant tumors in females (cervical, endometrial and epithelial ovarian cancer), and it is a serious threat to women's health (8). The development of epithelial ovarian cancer is a multi-factor and multi-step process and is not only associated with genetic changes, but also epigenetic changes. The role of epigenetic changes has become more apparent. DNA methylation is an important epigenetic mechanism. In recent years, it has been considered as the third mechanism which inactivates tumor suppressor genes, as well as loss of heterozygosity and gene mutation (9). A related study found that P16, ARHI, FHIT and other tumor suppressor gene promoter $\mathrm{CpG}$ islands have overmethylation in epithelial ovarian cancer (10-12). Makarla et al (13) analyzed the methylation status of the promoters of 8 tumor-related suppressor genes in epithelial ovarian tumors, including RASSF1A and P16, using the MSP method and found that all 8 genes had methylation in invasive carcinoma, but only two genes were methylated in benign ovarian tumor tissue. This indicates that the abnormal methylation of genes in ovarian cancer is a common phenomenon and suggests that abnormal DNA methylation may play an important role in the development of ovarian carcinoma in humans.

The WWOX gene is a newly identified tumor suppressor gene. It was first isolated and identified with shotgun sequencing by Bednarek et al (4) in 2000 and plays an important role in promoting cell apoptosis and inhibiting cell proliferation, adhesion and transfer. Our previous studies have shown that the expression of WWOX mRNA and WWOX protein in epithelial ovarian cancer tissues and cell line were clearly reduced $(14,15)$. To further explore the correlation between the methylation state of WWOX gene promoter $\mathrm{CpG}$ islands and epithelial ovarian cancer, we used the MSP method to detect the methylation of $\mathrm{CpG}$ islands of the WWOX gene in different types of ovarian cancer. The findings confirmed that the WWOX gene was methylated in ovarian epithelial cancer. Combined with previous studies, we speculate that the aberrant methylation of $\mathrm{CpG}$ island may lead to reduced or absent WWOX gene expression, which may be an important mechanism for the occurrence of epithelial ovarian cancer.

It is widely believed that the abnormal methylation of DNA often occurs in the early stage of cancer. In our study, the methylation rate of WWOX gene promoter $\mathrm{CpG}$ islands in borderline epithelial ovarian tumors was higher than that of normal ovarian tissue and ovarian benign epithelial tumor and in the one case of benign ovarian epithelial tumor the region was completely methylated. These are indications that molecular level changes may already exist in ovarian epithelial cells before the appearance of the cancer. The results also illustrate that abnormally methylated DNA may be an early event in carcinogenesis involved in the occurrence of epithelial ovarian cancer. In addition, the results show that the rate of $\mathrm{CpG}$ island methylation in WWOX gene promoter region in late-stage epithelial ovarian cancer tissues was higher than that of early-stage epithelial ovarian cancer tissues. We speculate that the reduction or absence of expression of the WWOX gene caused by unusual promoter methylation may be closely correlated with the clinical development of epithelial ovarian cancer.

As an important epigenetic mechanism, DNA methylation is currently the subject of intense research. This study shows that the aberrant methylation state of WWOX gene $\mathrm{CpG}$ islands is closely correlated with the occurrence and development of epithelial ovarian cancer. In the future, detecting DNA methylation of the WWOX gene may become a new technique to determine the occurrence, development and prognosis of epithelial ovarian cancer. In addition, our findings imply that aberrant DNA methylation induced WWOX gene inactivation, which led to the occurrence of epithelial ovarian cancer. However, whether epithelial ovarian cancer progression also causes WWOX gene methylation, or if there is a combination of the two mechanisms, requires further study.

\section{References}

1. Li XQ, Guo YY, De W: DNA methylation and microRNAs in cancer. World J Gastroenterol 18: 882-888, 2012.

2. Nagarajan RP and Costello JF: Epigenetic mechanisms in glioblastoma multiforme. Semin Cancer Biol 19: 188-197, 2009.

3. Kanai Y: Alterations of DNA methylation and clinicopathological diversity of human cancers. Pathol Int 58: 544-558, 2008.

4. Bednarek AK, Laflin KJ, Daniel RL, et al: WWOX, a novel WW domain-containing protein mapping to human chromosome 16q23.3-24.1, a region frequently affected in breast cancer. Cancer Res 60: 2140-2145, 2000.

5. Iliopoulos D, Guler G, Han SY, et al: Fragile genes as biomarkers: epigenetic control of WWOX and FHIT in lung, breast and bladder cancer. Oncogene 24: 1625-1633, 2005.

6. Qin HR, Iliopoulos D, Semba S, et al: A role for the WWOX gene in prostate cancer. Cancer Res 66: 6477-6481, 2006.

7. Yan J, Zhang M, Zhang J, et al: Helicobacter pylori infection promotes methylation of WWOX gene in human gastric cancer. Biochem Biophys Res Commun 408: 99-102, 2011.

8. Roett MA and Evans P: Ovarian cancer: an overview. Am Fam Physician 80: 609-616, 2009.

9. Azhikina TL and Sverdlov ED: Study of tissue-specific CpG methylation of DNA in extended genomic loci. Biochemistry (Mosc) 70: 596-603, 2005.

10. Surowiak P, Materna V, Maciejczyk A, et al: Decreased expression of p16 in ovarian cancers represents an unfavourable prognostic factor. Histol Histopathol 23: 531-538, 2008.

11. Feng W, Marquez RT, Lu Z, et al: Imprinted tumor suppressor genes ARHI and PEG3 are the most frequently down-regulated in human ovarian cancers by loss of heterozygosity and promoter methylation. Cancer 112: 1489-1502, 2008.

12. Potapova A, Hoffman AM, Godwin AK, et al: Promoter hypermethylation of PALB2 susceptibility gene in inherited and sporadic breast and ovarian cancer. Cancer Res 68: 998-1002, 2008.

13. Makarla PB, Saboorian MH, Ashfaq R, et al: Promoter hypermethylation profile of ovarian epithelial neoplasms. Clin Cancer Res 11: 5365-5369, 2005.

14. Yan HC, Lu XY and Han QY: WWOX mRNA expression in epithelial ovarian cancer and its clinical significance. Acta Academiae Medicinae Xuzhou 27: 126-128, 2007 (In Chinese).

15. Yan HC, Xue JQ, Lu XY, et al: Effects of WWOX gene transfection on cell growth of epithelial ovarian cancer. Zhonghua Fu Chan Ke Za Zhi 43: 361-365, 2008 (In Chinese). 